\title{
Coefficient estimates for starlike functions
}

\section{M.L. Mogra and O.P. Juneja}

Let $S_{k}^{*}(\alpha, \beta)$ denote the class of functions

$$
f(z)=z+\sum_{n=k+1}^{\infty} a_{n} z^{n}
$$

analytic in the unit disc $\Delta \equiv\{z:|z|<I\}$ and satisfying

$$
\left|\left(z \frac{f^{\prime}(z)}{f(z)}-1\right) /\left\{2 \beta\left(z \frac{f^{\prime}(z)}{f(z)}-\alpha\right)-\left[z \frac{f^{\prime}(z)}{f(z)}-1\right)\right\}\right|<1
$$

for some $\alpha, \beta \quad(0 \leq \alpha<1,0<\beta \leq 1)$ and for all $z \in \Delta$. In the present paper, sharp coefficient estimates for functions in $S_{k}^{*}(\alpha, \beta)$ have been obtained. The results thus obtained not only generalize the corresponding results of Thomas $H$. MacGregor (Michigan Math. J. 10 (1963), 277-281), A.V. Boyd (Proc. Amer. Math. Soc. 17 (1966), 1016-1018) and others, but also give rise to analogous results for various other subclasses of starlike functions.

\section{Introduction}

Let $f(z)$ be regular in the unit disc $\Delta \equiv\{z:|z|<1\}$ and normalized by the conditions $f(0)=0, f^{\prime}(0)=1$. The power series representation for such a function is

$$
f(z)=z+\sum_{n=2}^{\infty} a_{n} z^{n}
$$

Received 26 January 1977. 
If $f(z)$ satisfies the condition

$$
\operatorname{Re}\left\{z \frac{f^{\prime}(z)}{f^{\prime}(z)}\right\}>0
$$

for all $z \in \Delta$, then it is well known [9, p. 221] that (1.2) is both necessary and sufficient for $f$ to be univalent and starlike with respect to the origin in $\Delta$. For starlike functions the Bieberbach conjecture $\left|a_{n}\right| \leq n$ holds for all $n$, and equality occurs only for the functions $f(z)=z /(1+\varepsilon z)^{2}$, where $|\varepsilon|=1$. MacGregor [6] obtained upper bounds for the moduli of the coefficients of a starlike function whose power series representation in $\Delta$ is of the form

$$
f(z)=z+\sum_{n=k+1}^{\infty} a_{n} z^{n}
$$

Boyd [1] extended MacGregor's result to the class $S_{\alpha}^{*}$ of starlike functions of order $\alpha(0 \leq \alpha<1)$; that is, $f \in S_{\alpha}^{*}$ if it is univalent and $\operatorname{Re}\left(z f^{\prime}(z) / f(z)\right)>\alpha$ for $z \in \Delta$. Various other subclasses of starlike functions have been considered and corresponding coefficient estimates obtained for them by Singh [13, 14], Padmanabhan [10], Eenigenburg [2], McCarty [7], Mogra [8], and others.

Recently, the authors [4] introduced the class of starlike functions of order $\alpha \quad(0 \leq \alpha<1)$ and type $\beta \quad(0<\beta \leq 1)$ and made a preliminary study of its properties. Later, Gupta and Jain [3] obtained certain results for functions of this class when all $a_{n}$ are negative. These results were the analogues of the corresponding results obtained by Silverman [12] for starlike functions of order $\alpha$. However, to broaden the scope of applicability of the results obtained, the authors [5] have very recently modified the definition of starlike functions of order $\alpha$ $(0 \leq \alpha<1)$ and type $\beta \quad(0<\beta \leq 1)$, which is as follows.

DEFINITION. Let $f(z)=z+\sum_{n=2}^{\infty} a_{n} z^{n}$ be analytic in the unit disc $\Delta$. Then $f$ is said to be starlike of order $\alpha$ and type $\beta$, if it satisfies the condition

$$
\left|\left(z \frac{f^{\prime}(z)}{f(z)}-1\right\} /\left\{2 \beta\left\{z \frac{f^{\prime}(z)}{f(z)}-\alpha\right\}-\left(z \frac{f^{\prime}(z)}{f(z)}-1\right)\right\}\right|<1
$$


for some $\alpha, \beta \quad(0 \leq \alpha<1,0<\beta \leq 1)$ and $z \in \Delta$.

The class of starlike functions of order $\alpha(0 \leq \alpha<1)$ and type $\beta$ $(0<\beta \leq 1)$ we shall denote by $S^{*}(\alpha, \beta)$.

It can be easily seen that $S^{*}(\alpha, \beta)$ includes the subclasses of starlike functions for different values of the parameters $\alpha$ and $\beta$. Hence a study of its various properties leads to a unified study of these subclasses. In the present note, we determine sharp coefficient estimates for the class $S_{k}^{*}(\alpha, \beta)$ of starlike functions of order $\alpha$ and type $\beta$ whose power series representation is of the form (1.3). The results thus obtained not only generalize the corresponding results of MacGregor [6], Boyd [1], and others, but also give rise to analogous results for the functions of the form (1.3) belonging to the classes introduced and studied by Singh [13, 14], Padmanabhan [10], Wright [15], McCarty [7], and Eenigenburg [2].

\section{Some coefficient estimates}

THEOREM. Let $f(z)=z+\sum_{n=k+1}^{\infty} a_{n} z^{n}$ be in $S_{k}^{*}(\alpha, \beta)$.

(a) If $\beta(1-\alpha)>k(1-\beta)$, Let $M=\left[\frac{\beta(1-\alpha)}{k(1-\beta)}\right]$. Then

$$
\left|a_{n}\right| \leq \frac{k}{(n-1)(m-I) !} \prod_{\mu=0}^{m-1}\left((2 \beta-1) \mu+\frac{2 \beta(1-\alpha)}{k}\right)
$$

for $m k+1 \leq n \leq(m+1) k, m=1,2, \ldots, M+1$ and

(2.2) $\left|a_{n}\right| \leq \frac{k}{(n-1)(M+1) !} \prod_{\mu=0}^{M+1}\left((2 \beta-1) \mu+\frac{2 \beta(1-\alpha)}{k}\right), n>(M+2) k$.

(b) If $\beta(1-\alpha) \leq k(1-\beta)$, then

$$
\left|a_{n}\right| \leq \frac{2 \beta(1-\alpha)}{n-1} \text { for } n \geq k+1 \text {. }
$$

The estimates in (2.1) are sharp for $n=m k+1, m=1,2, \ldots$, while the estimates in (2.3) are sharp for all $n$.

Proof. We employ the technique used by MacGregor [6]. Thus, let $f \in S_{k}^{*}(\alpha, \beta)$; then we have 


$$
h(z)=\frac{z f^{\prime}(z)-f(z)}{2 \beta\left[z f^{\prime}(z)-\alpha f(z)\right]-\left(z f^{\prime}(z)-f(z)\right)}
$$

where $h$ is regular in $\Delta$ and satisfies $|h(z)|<1$ in $\Delta$. Also the power series for $h(z)$ begins with $c_{k} z^{k}+c_{k+1} z^{k+1}+\ldots$. Equating cpefficients of the same powers on both sides of the equation

$$
z f^{\prime}(z)-f(z)=h(z)\left\{2 \beta\left(z f^{\prime}(z)-\alpha f(z)\right)-\left(z f^{\prime}(z)-f(z)\right)\right\}
$$

or

$$
\text { (2.5) } \begin{aligned}
\sum_{n=k+1}^{\infty} & (n-1) a_{n} z^{n} \\
= & \left\{c_{k} z^{k}+c_{k+1} z^{k+1}+\ldots\right\}\left\{2 \beta(1-\alpha) z+\sum_{n=k+1}^{\infty}((2 \beta-1) n+1-2 \alpha \beta) a_{n} z^{n}\right\},
\end{aligned}
$$

we obtain

$$
(n-1) a_{n}=2 \beta(1-\alpha) c_{n-1} \text { for } n=k+1, k+2, \ldots, 2 k \text {. }
$$

Since $|h(z)|<1$, it follows that $\sum_{n=k}^{\infty}\left|c_{n}\right|^{2} \leq 1$ and so

$$
\sum_{n=k}^{2 k-1}\left|c_{n}\right|^{2} \leq 1
$$

From (2.6) and (2.7), we find that

$$
\sum_{n=k+1}^{2 k}(n-1)^{2}\left|a_{n}\right|^{2} \leq 4 \beta^{2}(1-\alpha)^{2} .
$$

(2.5) can be rewritten in the form

(2.9) $\sum_{n=\hat{k}+1}^{p}(n-1) a_{n} z^{n}+\sum_{n=p+1}^{\infty} d_{n} z^{n}$

$$
=h(z)\left\{2 \beta(1-\alpha) z+\sum_{n=\hat{k}+1}^{p-k}((2 \beta-1) n+1-2 \alpha \beta) a_{n} z^{n}\right\} .
$$

Since (2.9) has the form $F(z)=h(z) G(z)$, where $|h(z)|<1$, it follows that

$$
\frac{1}{2 \pi} \int_{0}^{2 \pi}\left|F\left(r e^{i \phi}\right)\right|^{2} d \phi \leq \frac{1}{2 \pi} \int_{0}^{2 \pi}\left|G\left(r e^{i \phi}\right)\right|^{2} d \phi
$$


for each $r(0<r<1)$. Expressing (2.10) in terms of the coefficients in (2.9), we get

$$
\text { (2.11) } \begin{aligned}
\sum_{n=k+1}^{p}(n-1)^{2}\left|a_{n}\right|^{2} r^{2 n} & +\sum_{n=p+1}^{\infty}\left|d_{n}\right|^{2} r^{2 n} \\
& \leq 4 \beta^{2}(1-\alpha)^{2} r^{2}+\sum_{n=k+1}^{p-k}((2 \beta-1) n+1-2 \alpha \beta)^{2}\left|a_{n}\right|^{2} r^{2 n} .
\end{aligned}
$$

In particular, (2.11) implies

(2.12) $\sum_{n=k+1}^{p}(n-1)^{2}\left|a_{n}\right|^{2} r^{2 n}$

$$
\leq 4 \beta^{2}(1-\alpha)^{2} r^{2}+\sum_{n=\hat{k}+1}^{p-k}((2 \beta-1) n+1-2 \alpha \beta)^{2}\left|a_{n}\right|^{2} r^{2 n} .
$$

Letting $r \rightarrow 1$ in (2.12), we conclude that

(2.13) $\sum_{n=k+1}^{p}(n-1)^{2}\left|a_{n}\right|^{2} \leq 4 \beta^{2}(1-\alpha)^{2}+\sum_{n=k+1}^{p-k}((2 \beta-1) n+1-2 \alpha \beta)^{2}\left|a_{n}\right|^{2}$.

This inequality is equivalent to

$$
\text { (2.14) } \begin{aligned}
& \sum_{n=p-k+1}^{p}(n-1)^{2}\left|a_{n}\right|^{2} \\
& \leq 4 \beta^{2}(1-\alpha)^{2}+\sum_{n=k+1}^{p-k}\left\{((2 \beta-1) n+1-2 \alpha \beta)^{2}-(n-1)^{2}\right\}\left|a_{n}\right|^{2} .
\end{aligned}
$$

Now two cases arise.

(a) If $\beta(1-\alpha)>k(1-\beta)$, then by an inductive argument we will establish the inequalities

$$
\begin{aligned}
& \text { (2.15a) } \sum_{n=m k+1}^{(m+1) k}(n-1)^{2}\left|a_{n}\right|^{2} \leq\left\{\frac{k}{(m-1) !} \prod_{\mu=0}^{m-1}\left((2 \beta-1) \mu+\frac{2 \beta(1-\alpha)}{k}\right)\right\}^{2} \text {, } \\
& \text { (2.15b) } \sum_{n=m k+1}^{(m+1) k}\left\{((2 \beta-1) n+1-2 \alpha \beta)^{2}-(n-1)^{2}\right\}\left|a_{n}\right|^{2} \\
& \leq\left\{\frac{1}{m !} \prod_{\mu=0}^{m-1}\left\{(2 \beta-1) \mu+\frac{2 \beta(1-\alpha)}{k}\right)\right\}^{2}\left\{((2 \beta-1) m k+2 \beta(1-\alpha))^{2}-m^{2} k^{2}\right\},
\end{aligned}
$$

for $m=1,2, \ldots, M+1 ; M=\left[\frac{\beta(1-\alpha)}{k(1-\beta)}\right]$ where [p] denotes the greatest 
integer not greater than $p$.

For $m=1,(2.15 a)$ gives

$$
\sum_{n=k+1}^{2 k}(n-1)^{2}\left|a_{n}\right|^{2} \leq 4 \beta^{2}(1-\alpha)^{2}
$$

which is the same as (2.8). Thus $(2.15 a)$ is valid for $m=1$. We can prove (2.15b) for $m=1$ by using (2.8) as follows:

$$
\begin{aligned}
\sum_{n=k+1}^{2 k}\left\{((2 \beta-1) n+1-2 \alpha \beta)^{2}-(n-1)^{2}\right\}\left|a_{n}\right|^{2} & \\
& \leq \frac{[(2 \beta-1) k+2 \beta(1-\alpha)]^{2}-k^{2}}{k^{2}} \sum_{n=k+1}^{2 k}(n-1)^{2}\left|a_{n}\right|^{2} \\
& \leq \frac{4 \beta^{2}(1-\alpha)^{2}}{k^{2}}\left\{((2 \beta-1) k+2 \beta(1-\alpha))^{2}-k^{2}\right\} .
\end{aligned}
$$

Now suppose that $(2.15 a)$ and $(2.15 b)$ hold for $m=1,2, \ldots, q-1$. Using (2.14) with $p=(q+1) k$ and the inductive hypothesis concerning (2.15a), we obtain the inequalities

$$
\begin{aligned}
& \sum_{n}^{(q+1) k}(n-1)^{2}\left|a_{n}\right|^{2} \\
& \leq 4 \beta^{2}(1-\alpha)^{2}+\sum_{n=k+1}^{q k}\left\{((2 \beta-1) n+1-2 \alpha \beta)^{2}-(n-1)^{2}\right\}\left|a_{n}\right|^{2} \\
& =4 \beta^{2}(1-\alpha)^{2}+\sum_{m=1}^{q-1} n \sum_{m k+1}^{(m+1) k}\left\{((2 \beta-1) n+1-2 \alpha \beta)^{2}-\left.(n-1)^{2}|| a_{n}\right|^{2}\right. \\
& \leq 4 \beta^{2}(1-\alpha)^{2}+\sum_{m=1}^{q-1}\left\{\frac{1}{m !} \prod_{\mu=0}^{m-1}\left((2 \beta-1) \mu+\frac{2 \beta(1-\alpha)}{k}\right)\right\}^{2}\left\{((2 \beta-1) m k+2 \beta(1-\alpha))^{2}-m^{2} k^{2}\right\} \\
& =\left\{\frac{k}{(q-1) !} \prod_{\mu=0}^{q-1}\left((2 \beta-1)_{\mu}+\frac{2 \beta(1-\alpha)}{k}\right)\right\}^{2} .
\end{aligned}
$$

The last equality can be easily obtained by an inductive argument on $q$. This last sequence of inequalities implies (2.15a) where $m=q$. Continuing our argument, we use (2.15a) with $m=q$ to deduce (2.15b) for $m=q$ as follows: 
$(q+1) k$

$\sum_{n=q k+1}\left\{((2 \beta-1) n+1-2 \alpha \beta)^{2}-(n-1)^{2}\right\}\left|a_{n}\right|^{2}$

$$
\begin{aligned}
& \leq \frac{((2 \beta-1) q k+2 \beta(1-\alpha))^{2}-q^{2} k^{2}}{q^{2} k^{2}} \sum_{n=q k+1}^{(q+1) k}(n-1)^{2}\left|a_{n}\right|^{2} \\
& \leq \frac{((2 \beta-1) q k+2 \beta(1-\alpha))^{2}-q^{2} k^{2}}{q^{2} k^{2}}\left\{\frac{k}{(q-1) !} \prod_{\mu=0}^{q-1}\left\{(2 \beta-1) \mu+\frac{2 \beta(1-\alpha)}{k}\right)\right\}^{2} \\
& =\left\{\frac{1}{q !} \prod_{u=0}^{q-1}\left((2 \beta-1) \mu+\frac{2 \beta(1-\alpha)}{k}\right)\right\}^{2}\left\{((2 \beta-1) q k+2 \beta(1-\alpha))^{2}-q^{2} k^{2}\right\} .
\end{aligned}
$$

This completes the proof of (2.15a) and (2.15b). Now (2.1) follows from (2.15a).

To prove (2.2), suppose $n>(M+2) k$. Putting $p=(q+1) k$ in (2.14), we have

$$
\sum_{n=q k+1}^{(q+1) k}(n-1)^{2}\left|a_{n}\right|^{2} \leq 4 \beta^{2}(1-\alpha)^{2}+\sum_{n=k+1}^{q k}\left\{((2 \beta-1) n+1-2 \alpha \beta)^{2}-(n-1)^{2}\right\}\left|a_{n}\right|^{2} .
$$

Hence, for $n>(M+2) k$, we have

(2.16) $(n-1)^{2}\left|a_{n}\right|^{2}$

$$
\begin{aligned}
\leq 4 \beta^{2}(1-\alpha)^{2} & +\sum_{n=k+1}^{q k}\left\{((2 \beta-1) n+1-2 \alpha \beta)^{2}-(n-1)^{2}\right\}\left|a_{n}\right|^{2} \\
=4 \beta^{2}(1-\alpha)^{2} & +\sum_{n=k+1}^{(M+2) k}\left\{((2 \beta-1) n+1-2 \alpha \beta)^{2}-(n-1)^{2}\right\}\left|a_{n}\right|^{2} \\
& +\sum_{n=(M+2) k+1}^{q k}\left\{((2 \beta-1) n+1-2 \alpha \beta)^{2}-(n-1)^{2}\right\}\left|a_{n}\right|^{2} \\
=4 \beta^{2}(1-\alpha)^{2} & +\sum_{m=1}^{M+1} \sum_{n=m k+1}^{(m+1) k}\left\{((2 \beta-1) n+1-2 \alpha \beta)^{2}-(n-1)^{2}\right\}\left|a_{n}\right|^{2} \\
& +\sum_{m=11+2}^{q-1} \sum_{n=m k+1}^{(m+1) k}\left\{((2 \beta-1) n+1-2 \alpha \beta)^{2}-(n-1)^{2}\right\}\left|a_{n}\right|^{2} \\
\leq 4 \beta^{2}(1-\alpha)^{2} & +\sum_{m=1}^{M+1} \sum_{n=m k+1}^{(m+1) k}\left\{((2 \beta-1) n+1-2 \alpha \beta)^{2}-(n-1)^{2}\right\}\left|a_{n}\right|^{2} .
\end{aligned}
$$

Using (2.15a) in (2.16), we obtain 


$$
(n-1)^{2}\left|a_{n}\right|^{2} \leq\left\{\frac{k}{(M+1) !} \prod_{\mu=0}^{M+1}\left((2 \beta-1) \mu+\frac{2 \beta(1-\alpha)}{k}\right)\right\}^{2}
$$

that is,

$$
\left|a_{n}\right| \leq \frac{k}{(n-1)(M+1) !} \prod_{\mu=0}^{M+1}\left((2 \beta-1)_{\mu}+\frac{2 \beta(1-\alpha)}{k}\right), n>(M+2) k .
$$

This proves (2.2).

(b) If $\beta(1-\alpha) \leq k(1-\beta)$, then (2.13) gives

$$
\sum_{n=k+1}^{p}(n-1)^{2}\left|a_{n}\right|^{2} \leq 4 \beta^{2}(1-\alpha)^{2}
$$

or

$$
(n-1)^{2}\left|a_{n}\right|^{2} \leq 4 \beta^{2}(1-\alpha)^{2} \quad \text { if } n \geq k+1
$$

that is,

$$
\left|a_{n}\right| \leq \frac{2 \beta(1-\alpha)}{n-1} \text { if } n \geq k+1 \text {, }
$$

which gives (2.3).

The function $f$, given by

$$
z \frac{f^{\prime}(z)}{f(z)}=\frac{1-(2 \alpha \beta-1) z^{k}}{1-(2 \beta-1) z^{k}} \text { where } \beta(1-\alpha)>k(1-\beta),
$$

shows that the estimates in (2.1) are sharp for $n=m k+1$, $m=1,2,3, \ldots$ while the estimates in (2.3) are sharp for the function

$$
f(z)=z \exp \left\{[2 \beta(1-\alpha) /(n-1)] z^{n-1}\right\},
$$

where $\beta(1-\alpha) \leq k(1-\beta)$ and $n \geq k+1$.

Putting $\beta=1$ in the theorem, we get the following result due to Boyd [1].

COROLLARY 1. If $f(z)=z+\sum_{n=k+1}^{\infty} a_{n} z^{n}$ is starlike of order $\alpha$, $0 \leq \alpha<1$, then 


$$
\left|a_{n}\right| \leq \frac{k}{(n-1)(m-1) !} \prod_{\mu=0}^{m-1}\left(\mu+\frac{2(1-\alpha)}{k}\right)
$$

where $m k+1 \leq n \leq(m+1) k, m=1,2, \ldots$. The result is sharp for $n=m k+1, m=1,2, \ldots$ for the function

$$
f(z)=\frac{z}{\left(1-z^{k}\right)^{2(1-\alpha) / k}} .
$$

The following result, due to MacGregor [6], can be obtained by taking $(\alpha, \beta)=(0,1)$ in the theorem.

$$
\begin{aligned}
& \text { COROLLARY 2. If } f(z)=z+\sum_{n=k+1}^{\infty} a_{n} z^{n} \text { is in } s_{k}^{*}(0,1) \text {, then } \\
& \qquad a_{n} \mid \leq \frac{k}{(n-1)(m-1) !} \prod_{\mu=0}^{m-1}(\mu+(2 / k))
\end{aligned}
$$

where $m k+1 \leq n \leq(m+1) k, m=1,2, \ldots$. The estimates are sharp for $n=m k+1 \quad(m=1,2, \ldots)$, for

$$
f(z)=\frac{z}{\left(1-z^{k}\right)^{2 / k}} .
$$

REMARKS. (i) The coefficient estimates determined in [5] for the starlike functions of order $\alpha$ and type $\beta$ can be obtained by putting $k=1$ in the theorem.

(ii) Putting

$$
\begin{aligned}
\beta & =\frac{1}{2}, \\
(\alpha, \beta) & =\left(0, \frac{1}{2}\right) ; \\
(\alpha, \beta) & =\left(0,(2 \delta-1) / 2 \delta \text { where } \delta>\frac{1}{2}\right) ; \\
(\alpha, \beta) & =((1-\gamma) /(1+\gamma),(1+\gamma) / 2)
\end{aligned}
$$

where $0<\gamma \leq 1$, and replacing $\alpha$ by $1-\alpha$ and $\beta$ by $\frac{1}{2}$ in the theorem, we get respectively the corresponding coefficient estimates for the functions of the form (1.3) belonging to the classes introduced by McCarty [7], Singh [13, 14], Padmanabhan [10], and Eenigenburg [2].

(iii) The results due to Schild [11], Singh [13, 14], Eenigenburg [2], Mogra [8], McCarty [7], can be obtained by taking different values of the parameters $\alpha, \beta \quad(0 \leq \alpha<1,0<\beta \leq 1)$ with $k=1$ in the theorem. 


\section{References}

[1] A.V. Boyd, "Coefficient estimates for starlike functions of order $\alpha$ ", Proc. Amer. Math. Soc. 17 (1966), 1016-1018.

[2] P.J. Eenigenburg, "A class of starlike mappings in the unit disc", Compositio Math. 24 (1972), 235-238.

[3] V.P. Gupta and P.K. Jain, "Certain classes of univalent functions with negative coefficients", Bull. Austral. Math. Soc. 14 (1976), 409-416.

[4] O.P. Juneja and M.L. Mogra, "On starlike functions of order $\alpha$ and type B ", Notices Amer. Math. Soc. 22 (1975), A-384; Abstract No. $75 \mathrm{~T}-\mathrm{B} 80$.

[5] O.P. Juneja and M.L. Mogra, "On starlike functions of order $\alpha$ and type B ", Rev. Roumaine Math. Pures Appl. (to appear).

[6] Thomas H. MacGregor, "Coefficient estimates for starlike mappings", Michigan Math. J. 10 (1963), 277-281.

[7] Carl P. McCarty, "Starlike functions", Proc. Amer. Math. Soc. 43 (1974), 361-366.

[8] M.L. Mogra, "On a class of starlike functions in the unit disc I", J. Indian Math. Soc. (to appear).

[9] Zeev Nehari, Conformal mapping (McGraw-Hill, New York, Toronto, London, 1952).

[10] K.S. Padmanabhan, "On certain classes of starlike functions in the unit disk", J. Indian Math. Soc. (N.S.) 32 (1968), 89-103.

[11] Albert Schild, "On starlike functions of order $\alpha$ ", Amer. J. Math. 87 (1965), 65-70.

[12] Herb Silverman, "Univalent functions with negative coefficients", Proc. Amer. Math. Soc. 51 (1975), 109-116.

[13] Ram Singh, "On a class of star-like functions", Compositio Math. 19 (1968), 78-82.

[14] Ram Singh, "On a class of starlike functions. II", Ganita 19 (1968), 103-110. 
[15] D.J. Wright, "On a class of starlike functions", Compositio Math. 21 (1969), 122-124.

Department of Mathematics,

Indian Institute of Technology Kanpur,

Kanpur,

India. 